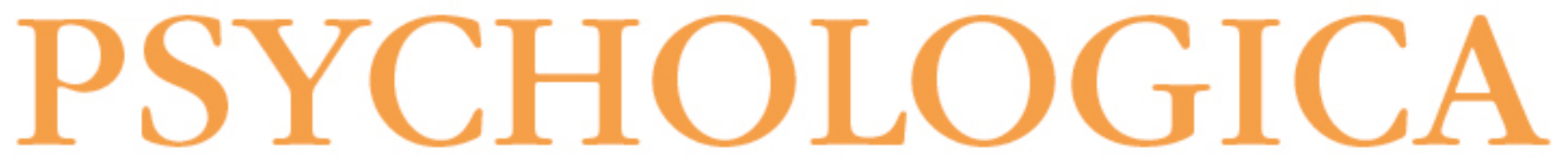

Ciclo vital da família: reflexão sobre as especificidades étnicas e culturais do desenvolvimento das famílias africanas

Autor(es): $\quad$ Simões, Tchilissila Alicerces; Alberto, Isabel M.

Publicado por: Imprensa da Universidade de Coimbra

URL

persistente: URI:http://hdl.handle.net/10316.2/42367

DOI: DOI:https://doi.org/10.14195/1647-8606_60-1_5

Accessed : $\quad$ 26-Apr-2023 10:01:05

A navegação consulta e descarregamento dos títulos inseridos nas Bibliotecas Digitais UC Digitalis, UC Pombalina e UC Impactum, pressupõem a aceitação plena e sem reservas dos Termos e Condições de Uso destas Bibliotecas Digitais, disponíveis em https://digitalis.uc.pt/pt-pt/termos.

Conforme exposto nos referidos Termos e Condições de Uso, o descarregamento de títulos de acesso restrito requer uma licença válida de autorização devendo o utilizador aceder ao(s) documento(s) a partir de um endereço de IP da instituição detentora da supramencionada licença.

Ao utilizador é apenas permitido o descarregamento para uso pessoal, pelo que o emprego do(s) título(s) descarregado(s) para outro fim, designadamente comercial, carece de autorização do respetivo autor ou editor da obra.

Na medida em que todas as obras da UC Digitalis se encontram protegidas pelo Código do Direito de Autor e Direitos Conexos e demais legislação aplicável, toda a cópia, parcial ou total, deste documento, nos casos em que é legalmente admitida, deverá conter ou fazer-se acompanhar por este aviso.

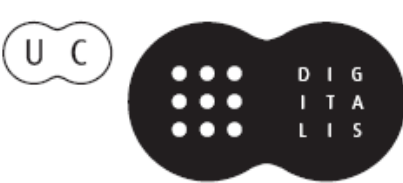


vOLUME
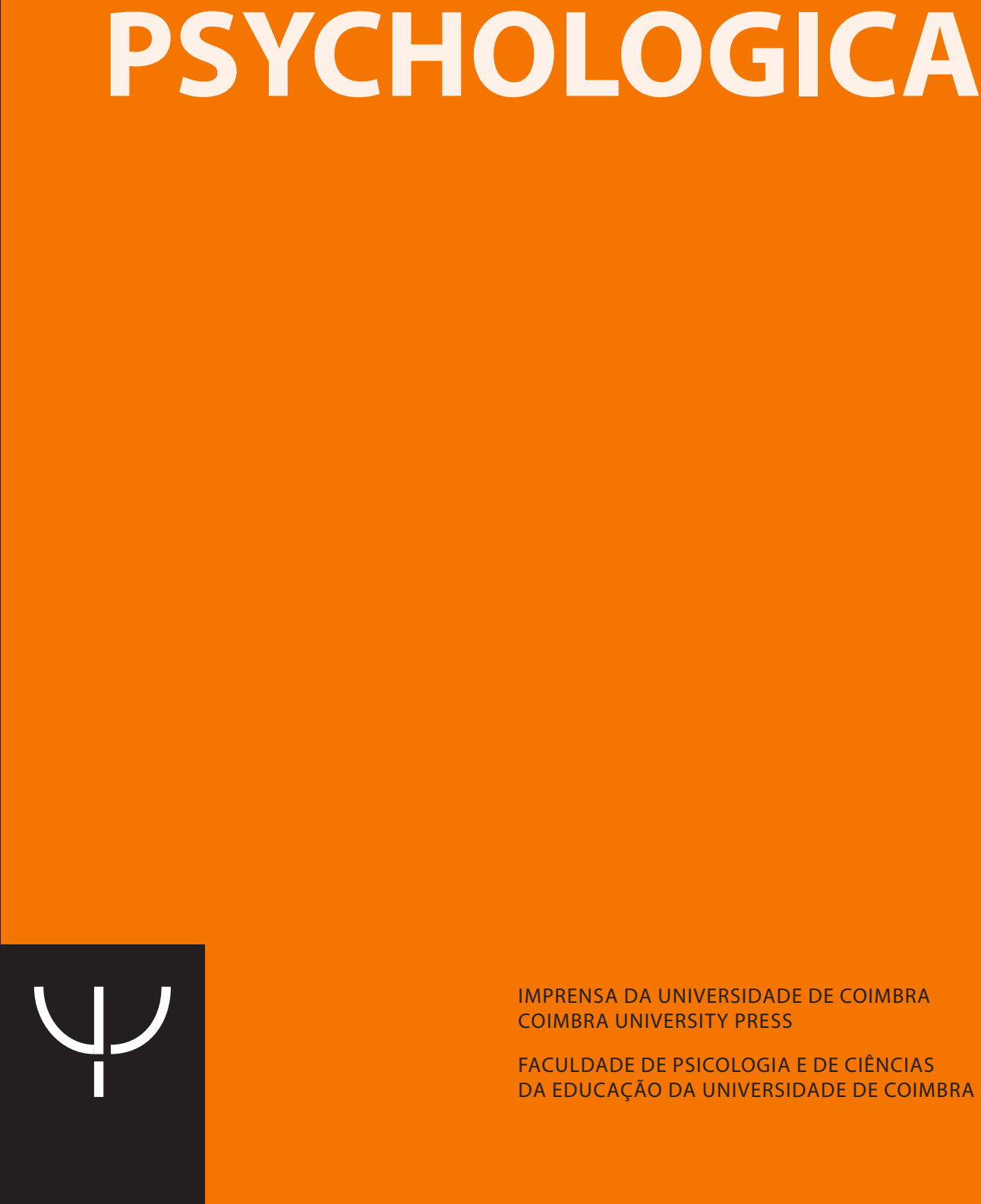

IMPRENSA DA UNIVERSIDADE DE COIMBRA

COIMBRA UNIVERSITY PRESS

FACULDADE DE PSICOLOGIA E DE CIÊNCIAS

DA EDUCAÇÃO DA UNIVERSIDADE DE COIMBRA 


\title{
Ciclo vital da família: Reflexão sobre as especificidades étnicas e culturais do desenvolvimento das famílias africanas
}

\author{
Tchilissila Alicerces Simões ${ }^{1}$ e Isabel M. Alberto ${ }^{2}$
}

Family life cycle: Reflections on ethnic and cultural specificities of African's family development

\begin{abstract}
Family psychology has emphasized the role of ethnic and cultural specifics in family development and functioning. However, little is known about how African (sub-Saharan) families function over the course of their development. This paper aims to identify in which way the theoretical models and empirical studies characterise the development and functioning (in terms of stress/tension, strengths, rituals and routines) of African families during their life cycle. The existing theory was revised based on bibliographic research, leading to the analysis of 22 articles. The results illustrate a lack of research focused on African families. The researches mentioned in the analysed articles show that there are specific issues related to triggering situations of stress/tension and strengths within African families, as well as the stabilizing and organizational role of rituals and routines during transitional times. From the theoretical revision emerged the need to create models of family development that are adjusted to the ethnic and cultural specificities of these families, as well as to promote further research into its functioning,
\end{abstract}

1 Doutoranda em Psicologia clínica, Faculdade de Psicologia e de Ciências da Educação da Universidade de Coimbra. Email: tchilissila@gmail.com

2 Professora Auxiliar da Faculdade de Psicologia e de Ciências da Educação da Universidade de Coimbra, PhD em Psicologia. Membro do CENCIFOR. Email: isamaria@fpce.uc.pt

Artigo recebido a 11-04-2016 e aprovado a 04-10-2016. 
so as to create a thorough theoretical framework that can be applied to evaluate and intervene on a family level.

Keywords: family life cycle; family stress/tension; family strengths; family rituals; family routines

\section{Resumo}

A Psicologia da Família tem enfatizado o papel das especificidades étnicas e culturais no desenvolvimento e funcionamento familiar. No entanto, pouco se sabe sobre a forma como as famílias africanas (subsarianas) funcionam ao longo do seu curso desenvolvimental. Este trabalho pretende identificar de que modo os modelos teóricos e os estudos empíricos caracterizam o desenvolvimento e o funcionamento (ao nível dos stress/tensões, forças, rituais e rotinas) das famílias africanas ao longo do percurso vital. Para tal realizou-se uma revisão teórica sustentada pela pesquisa em bases bibliográficas, da qual resultou a análise de 22 artigos. Os resultados evidenciam a escassez de investigação focada nas famílias africanas. As pesquisas relatadas nos artigos analisados demonstram a existência de especificidades a nível das situações indutoras de stress/tensões e de forças das famílias africanas e o papel estabilizador e organizador dos rituais e das rotinas durante os momentos de transição familiar. Da revisão teórica emergiu a necessidade de se conceberem modelos do percurso de desenvolvimento familiar ajustado às especificidades étnicas e culturais destas famílias e de promover mais investigação sobre o seu funcionamento familiar, que permitam criar um quadro teórico e compreensivo válido para a avaliação e intervenção ao nível do sistema familiar.

Palavras-chave: ciclo vital da família; stress/tensões familiares; forças familiares; rituais familiares; rotinas familiares

\section{INTRODUÇÃO}

O conceito de ciclo vital surgiu no princípio do século XX com o intuito de traduzir o processo de desenvolvimento de organizações, instituições e famílias numa perspetiva de etapas e transições (O’Rand \& Krecker, 1990), destacando a evolução em espiral envolvendo as diferentes gerações (Asen \& Tomson, 1997; Neighbour, 1985).

A evolução familiar no ciclo vital desenrola-se num contexto ecológico que influencia a organização e os processos familiares que se desenvolvem ao longo 
do tempo em função da classe, religião, background geográfico, etnicidade [i.e., caraterísticas que determinados povos têm em comum, enquadrando a raça, a religião, as crenças e práticas baseadas na nacionalidade] e referências culturais [i.e., valores e papéis familiares, padrões de comunicação, estilos afetivos e padrões de interação interpessoal] (Hill, Murry, \& Anderson, 2005; McGoldrick, 1993, 1995; McGoldrick, Giordano, \& Garcia-Preto, 2005). Nesta revisão iremos utilizar os conceitos de etnicidade e cultura como sinónimos (McGoldrick, 1995; McGoldrick et al., 2005).

Entre os diversos grupos étnicos encontram-se diferenças relativamente à conceção de família no que concerne à inclusão ou não da família alargada, aos momentos que pontuam as fases de transição do ciclo vital e as respetivas tarefas desempenhadas em cada fase, aos rituais e cerimónias que assinalam essas transições, às forças e ao suporte que as famílias têm para lidar com o stress/tensões e os desafios que surgem ao longo das etapas (McGoldrick, 1995).

Nesse sentido, realizou-se uma revisão da literatura com o intuito de analisar a informação resultante dos estudos empíricos sobre o desenvolvimento e o funcionamento (i.e., stress/tensões, forças, rituais e rotinas) das famílias africanas ao longo do seu percurso de vida. O presente estudo surgiu do contacto dos autores com duas realidades distintas, mas que levantavam as mesmas questões: a existência de famílias africanas que emigram dos PALOP para Portugal, e a interação cada vez maior de profissionais e investigadores portugueses com contextos africanos, particularmente o angolano.

\section{DESENVOLVIMENTO DA FAMÍLIA: OS MODELOS DO CICLO VITAL DA FAMÍLIA}

As investigações baseadas no conceito de ciclo vital começaram com os trabalhos de Rowntree e Loomis (citado em Aldous, 1990) nos Estados Unidos da América que, ao estudarem a pobreza na comunidade americana, constataram que as famílias apresentam maior vulnerabilidade em fases de transição.

Nos países ocidentais surgiram diversos modelos compreensivos do ciclo vital da família, desde as propostas de Duvall, Hill e Rodgers (citado em Relvas, 1996) e Carter e McGoldrick (1995) nos Estados Unidos da América e de Relvas (1996) em Portugal. Estes modelos diferem quanto ao número de etapas e à sequência dos momentos de transição familiar (Widmer \& Gauthier, 2013), no entanto, todos defendem que o desenvolvimento familiar se caracteriza por crises (stress/ tensões) que o sistema tem de enfrentar, pelas forças familiares que constituem 
recursos para lidar com essas crises, bem como pelos rituais e as rotinas que são particulares a cada etapa do ciclo vital. No entanto, estes modelos foram construídos tendo como referência as famílias brancas nucleares intactas da classe média do Ocidente e é questionada a sua adequação para outros contextos culturais (Carter \& McGoldrick, 1995; Relvas, 1996; Shange, 2010; Widmer \& Gauthier, 2013).

\section{FUNCIONAMENTO FAMILIAR: STRESS/TENSÕES E DESAFIOS}

Ao longo do seu desenvolvimento, o sistema familiar passa por crises normativas (i.e., o casamento, a transição para a parentalidade, a adolescência dos filhos, a saída dos filhos adultos, o envelhecimento dos ascendentes e dos próprios membros do casal) ou inesperadas (e.g., morte de um filho, doença, desemprego) que alteram de forma significativa o funcionamento familiar, a nível das rotinas e dos papéis que os seus membros desempenham (Hoffman, 1995). Os rituais e as forças familiares constituem-se recursos do sistema familiar para enfrentar, lidar e superar essas crises (Boss, 2002; McCubbin \& Patterson, 1983).

Olson et al. (1989) identificaram os acontecimentos indutores de stress e tensões mais referenciados na pesquisa empírica e agruparam-nos em categorias, nomeadamente: Tensões intrafamiliares, tensões financeiras, tensões relacionadas com a conciliação entre o trabalho e a família, doenças, tensões conjugais, gravidezes não esperadas, transições familiares (tensões associadas aos movimentos de entrada e saída da família) e tensões pelas perdas de parentes. Estes autores defendem que existem situações de stress e tensões que têm incidência particular em determinadas etapas do ciclo vital da família; por exemplo, as tensões intrafamiliares (e.g., os conflitos entre o marido e a mulher) eram mais frequentes nas etapas "famílias com filhos em idade escolar" e "famílias com filhos adolescentes".

Assim, o stress/tensões familiares funcionam como um fator importante no funcionamento familiar ajustado (Boss, 2002; Lavee, 2013; Olson et al., 1989) e o conhecimento sobre as perceções e os significados que são atribuídos aos acontecimentos indutores de stress pelas famílias etnicamente diversas, nomeadamente as africanas, permite uma maior compreensão das respostas do sistema familiar a essas crises (Boss, 2002).

No entanto, como é consensual na literatura (Boss, 2002; Lavee, 2013; Melo \& Alarcão, 2011; Olson et al., 1989), a interferência do stress/tensões no funcionamento e na organização familiares é mediada pela influência das forças/recursos que a família tem ou julga ter. 


\section{FUNCIONAMENTO FAMILIAR: FORÇAS/ RECURSOS FAMILIARES}

Os estudos iniciais sobre as famílias negras, particularmente as afro-americanas, tendiam a focar-se nas suas disfuncionalidades e stress/tensões associadas a pobreza, a residência em bairros problemáticos e ao estatuto de minoria étnica (Hill et al., 2005). No entanto, nas últimas décadas tem-se registado uma crescente preocupação da comunidade científica e dos profissionais com a identificação das forças/ recursos destas famílias (Marks et al., 2008).

Boss (2002) defende que, perante uma situação indutora de stress, o sistema familiar ativa um conjunto de recursos individuais e familiares (e.g., a estabilidade financeira, rede de apoio familiar e social, cooperação entre os membros da família, flexibilidade, comunicação) para lidar com os desafios normativos ou inesperados que surgem. É ainda realçada a influência do enquadramento étnico/cultural no tipo de recursos que as famílias possuem e no modo como lidam com os acontecimentos indutores de stress (Boss, 2002; Melo \& Alarcão, 2011).

A análise das forças/recursos familiares no presente estudo resulta da necessidade de verificar se o pressuposto da "universalidade das forças familiares" (i.e., afeto e apreciação, o compromisso, a comunicação positiva, o "passar juntos tempos agradáveis”, o bem-estar espiritual, e a habilidade para lidar com as situações indutoras de stress e momentos de crise de forma efetiva) defendido pela literatura é identificado nos estudos com famílias africanas (cf., Defrain, 1999).

\section{FUNCIONAMENTO FAMILIAR: RITUAIS E ROTINAS}

A literatura evidencia o papel fundamental dos rituais e das rotinas como recursos que permitem à família lidar e superar as crises, sejam elas normativas ou inesperadas, garantindo um funcionamento familiar ajustado (Fiese et al., 2002).

Os conceitos de rituais e rotinas têm sido utilizados, ao nível da literatura, como se fossem sinónimos (Fiese et al., 2002; Lind, 2008). No entanto, enquanto os rituais se referem às celebrações imbuídas de significado que permitem o delineamento dos momentos de transição familiar de uma etapa de desenvolvimento para a seguinte (e.g., casamento), as rotinas integram os padrões repetitivos e previsíveis que caracterizam a interação quotidiana da unidade familiar, providenciando um sentido de continuidade, previsibilidade e segurança ao longo do tempo, e permitindo o ajuste das tarefas e funções dos membros do sistema familiar em cada etapa do ciclo vital (Bennett, Wolin, \& McAvity, 1988; Churchill \& Stoneman, 2004). 
Os rituais e as rotinas vão-se transformando ao longo do desenvolvimento familiar, sobretudo as rotinas, uma vez que se vão tornando mais consistentes e padronizadas ao longo da trajetória de vida familiar (Boss, 2002; Fiese et al., 2002; Relvas, 1996).

O presente estudo pretende complementar a revisão da literatura realizada por Fiese et al. (2002) sobre os rituais e rotinas familiares, procurando identificar nos estudos realizados com famílias africanas se há e quais são as particularidades a nível dos rituais e das rotinas destas famílias ao longo do seu percurso evolutivo. Interessa compreender qual é o papel que estas variáveis desempenham ao longo do ciclo vital da família, que permitem garantir a estabilidade da família sem comprometer a mudança e a evolução inerentes ao percurso desenvolvimental (Bennett et al., 1988; Fiese et al., 2002).

Tendo por base o objetivo do estudo e a informação que resultou da pesquisa teórica, estruturou-se a seção dos resultados em quatro tópicos principais: a) estudos empíricos sobre o ciclo vital da família; b) stress/tensões familiares; c) forças familiares; d) rituais familiares; e e) rotinas familiares.

\section{MÉTODO}

\section{Seleção das referências bibliográficas}

Considerando o objetivo do estudo, foi feita pesquisa de artigos qualitativos e quantitativos publicados no período entre 1960 e 2015, em bases de dados eletrónicas (EBSCO HOST, PROQUEST, PsycINFO, Scielo), livros editados, manuscritos não publicados (i.e., dissertações de mestrado e teses de doutoramento) e materiais referenciados nos artigos previamente identificados, usando como palavras-chave (em português e em inglês): etnicidade, cultura, África, famílias negras, famílias africanas, ciclo vital da família, desenvolvimento familiar, stress/tensões familiares, forças familiares, rituais e rotinas familiares.

A seleção dos artigos a incluir tinha por base os seguintes critérios: 1) Abordarem o ciclo vital de famílias africanas; 2) avaliarem o funcionamento familiar em pelo menos um dos tópicos - stress/tensões, forças, rituais e rotinas - em famílias africanas; 3) estarem escritos num idioma que as autoras dominassem (i.e., inglês, português, espanhol ou francês).

Da pesquisa realizada identificaram-se 26 artigos dos quais 22 foram incluídos nesta revisão. Os restantes foram excluídos pelos seguintes motivos: a) Tinham como 
foco o ciclo vital de famílias reconstituídas brasileiras e famílias chinesas inférteis (Jiang, Guo, Li, \& Feldman, 2013; Kauffmann, 2000); b) utilizavam a abordagem do desenvolvimento familiar integrada no âmbito da economia e, especificamente, do marketing (Van Rooyen \& Du Plessis, 2003); c) encontravam-se escritos em línguas não dominadas pelas autoras (AJ, 1998). Foram incluídos os estudos que, apesar de não envolverem amostras africanas, integravam amostras afro-americanas.

\section{Descrição das metodologias dos artigos selecionados}

Os estudos empíricos que se versaram sobre o ciclo vital da família utilizaram dados dos censos demográficos (Locoh, 1981) e genogramas (Hines, 1995).

Os estudos empíricos sobre o funcionamento familiar recorreram à recolha do relato das experiências pessoais através de entrevistas semiestruturadas, diários de observação e instrumentos de autorrelato, com uma reduzida percentagem (9\%) a apresentar a conjugação de medidas qualitativas e quantitativas.

O recurso a uma amostra da população geral recrutada pelo método de amostragem por conveniência caracterizou 36\% dos estudos revistos, exigindo, deste modo, cautela na interpretação dos resultados. Dos 22 artigos analisados, quatro integravam amostras de famílias afro-americanas, de condição socioeconómica

pobre e residentes em contexto urbano e cinco não descreveram as caraterísticas socioeconómicas das suas amostras.

\section{RESULTADOS E DISCUSSÃO}

O objetivo desta revisão teórica foi explorar e identificar a caracterização do desenvolvimento e o funcionamento (especificamente ao nível do stress/tensões, forças, rituais e rotinas) das famílias africanas ao longo do percurso vital, presente na literatura.

\section{Estudos sobre o ciclo vital das famílias africanas}

Da revisão feita, apenas dois artigos, o de Locoh (1981) e de Hines (1995), propõem um modelo do ciclo vital para as famílias negras. Locoh (1981) avalia a evolução familiar em países africanos de língua inglesa e francesa (cf., Tabela 1) através da análise estatística das taxas de nupcialidade, de natalidade, de fertilidade 
e de mortalidade, tendo verificado dois percursos de desenvolvimento familiar: Um percurso inclui quatro etapas, nomeadamente a I) maternidade - até aos cinco anos do último filho; II) mulheres casadas com filhos em crescimento; III) mulheres casadas sem filhos; e IV) viuvez ou dissolução do casamento. O segundo percurso constitui-se também por quatro etapas mas com aspetos distintos, designadamente: I) maternidade - até aos cinco anos do último filho; II) mulheres casadas com filhos em crescimento - 5 aos 18 anos; III) viuvez com filhos em crescimento a precisarem de cuidados; IV) viuvez sem filhos em crescimento a precisarem de cuidados.

Por sua vez, Hines (1995) estudou o desenvolvimento das famílias afro-americanas pobres, caracterizando-o como um ciclo "truncado", organizado em três etapas: 1) Adolescência/ Jovem adulto solteiro, que decorre entre os 10 e os 11 anos dos filhos e tem como tarefas a diferenciação do eu, o estabelecimento do eu no trabalho e o desenvolvimento de relacionamentos com os iguais; 2) Família com filhos que começa, para a maior parte das mulheres afro-americanas, sem um sistema de casal estabelecido, caracterizando-se pelo destaque dos papéis parentais. Verifica-se a parentificação de um dos filhos para ajudar a mãe e o realinhamento dos relacionamentos com a família de origem; a formação do casal vem a concretizar-se mais tarde, já com a existência de filhos; 3) Família no estádio tardio da vida, onde não há registo do "ninho vazio", ou seja, o casal idoso não vive sozinho (cf., Tabela 1).

Num estudo qualitativo realizado na África do Sul, Shange (2010) pretendeu comparar o desenvolvimento familiar de duas mulheres, uma de 65 anos e outra de 19 anos, tendo como referência a trajetória evolutiva proposta por Carter e McGoldrick (1995) (cf., Tabela 1). Shange (2010) identificou diferenças relevantes ao nível da conquista da independência pessoal, da formação do casal e da promoção da autonomia dos filhos jovens adultos. O autor verificou ainda a ausência de um "ninho vazio" nos dois casos em estudo (cf., Tabela 1).

Outros estudos com amostras africanas (Francisco, 2012; Goede, 2012; Greef, 2000; Júlio, 2012) avaliam o funcionamento familiar, em particular o stress/ tensões, forças e rotinas familiares tendo como base conceptual os modelos do ciclo vital mais difundidos (Carter \& McGoldrick, 1995; Relvas, 1996) mas que, de acordo com Shange (2010) não parecem ser os mais adequados para analisar os sistemas familiares africanos.

Estudos sobre o stress/tensões familiares no ciclo evolutivo das famílias afro-americanas e africanas

Dos 22 artigos revistos, seis focavam-se nos acontecimentos stressores/tensões das famílias afro-americanas e africanas em diferentes fases do seu desenvolvimento, 
através de duas metodologias: 1) descrição das situações percebidas como eventos stressores; e 2) avaliação das perceções familiares sobre o índice de stress familiar.

Num estudo com 115 casais afro-americanos e 136 casais brancos de origem europeia, Timmer, Veroff, e Hatchet (1996), com recurso a uma metodologia mista, defendem que os casais recém-casados de origem africana apresentam um maior nível de stress derivado de dificuldades económicas e de uma gravidez pré-marital (cf., Tabela 1). Nesta fase de formação do casal, a gestão dos papéis de género tradicionais e o número reduzido de amigos em comum também são tidos como situações indutoras de stress (Amato, 2011). As famílias nas etapas com filhos na escola e com filhos adolescentes apresentam mais stress relacionado com a escola e com o contexto laboral (Goosby, Caldwell, Bellatorre, \& Jackson, 2012) (cf., Tabela 1).

Em dois estudos qualitativos com amostras afro-americanas, um realizado por Marks et al. (2008) e o outro realizado por Philips, Wilmoth, e Marks (2012) verificou-se que a conciliação e gestão entre o trabalho e a vida familiar, as tensões familiares, os problemas de comunicação, o papel de cuidador simultâneo de familiares mais novos e mais idosos, a necessidade de fornecer suporte para os elementos da família extensa, problemas financeiros, doença ou morte de um dos filhos e as questões de infidelidade/confiança funcionam como acontecimentos indutores de stress numa fase mais avançada da vida familiar (cf., Tabela 1).

A literatura realça que, independentemente do nível socioeconómico das famílias africanas (ou afro-americanas), o funcionamento e o percurso vital destas é caracterizado pela residência em bairros problemáticos e pelo contacto frequente com elementos da família alargada (Cichy, Stawski, \& Almeida, 2012; Hill et al., 2005; Timmer et al., 1996). Taylor, Chatters, Woodward, e Brown, (2013) defenderam que a frequente interação com os elementos da família alargada resulta numa maior tensão para os casais afro-americanos quando comparados com indivíduos de outros grupos étnicos (e.g., brancos e hispânicos). Contudo, nenhum dos estudos realizados em contexto americano compara os índices de stress familiar percebido pelas famílias em diferentes etapas do ciclo vital. Desta revisão, apenas um estudo realizado em Angola por Francisco (2012) procurou avaliar o efeito da fase de desenvolvimento familiar ao nível do stress familiar percebido (cf., Tabela 1). Numa pesquisa com 141 participantes e tendo por base conceptual o modelo do ciclo vital da família de autoria de Relvas (1996), Francisco (2012) verificou que os indivíduos que se encontravam na etapa "formação do casal" e "famílias com filhos pequenos" registaram um índice mais elevado de stress familiar. No entanto, estes dados devem ser analisados com alguma reserva, considerando que não se sabe se o modelo do ciclo vital que serviu de referência corresponde à realidade africana. 


\section{Estudos sobre as forças familiares no ciclo evolutivo das famílias}

afro-americanas e africanas

Relativamente à análise das forças familiares, encontraram-se quatro estudos realizados com famílias afro-americanas e dois com amostras de famílias sul-africanas em fases específicas do desenvolvimento familiar (e.g., formação do casal, família com filhos pequenos, filhos adolescentes).

Chadilha, Veroff, e Leber (1998) realizaram uma investigação com uma amostra multicultural (cf., Tabela 1), que destacou o papel da família alargada como condição de força e recurso na etapa de formação das famílias afro-americanas. Por sua vez, Taylor e Roberts (1996) verificam uma associação positiva entre o apoio da rede familiar e as boas práticas parentais de mães afro-americanas com filhos adolescentes (cf., Tabela 1). Marks et al. (2008) verificaram na sua amostra de casais afro-americanos que as famílias que se encontram nas fases intermédias do ciclo de desenvolvimento destacam a religiosidade, a estabilidade financeira, a união conjugal, a comunicação e o compromisso como forças familiares (cf., Tabela 1). De acordo com Taylor et al. (2013) a vivência da religiosidade, o apoio da família alargada e a estabilidade financeira apresentam-se como forças importantes em todas as etapas do desenvolvimento das famílias afro-americanas.

A família alargada assume uma dupla função para os indivíduos afro-americanos, já que funciona ao mesmo tempo como fonte de stress e como força familiar (Chichy et al., 2012; Timmer et al., 1996). A família alargada pode constituir-se como fonte de stress e tensão familiar pelo facto de a interação frequente entre os elementos da família facilitar o conflito aberto (e.g., discussões) relacionado com as obrigações familiares, com o desacordo em torno das práticas educativas dos filhos ou pelo "efeito de contágio", isto é, quando um dos elementos da família alargada tem um problema (e.g., doença de uma irmã, stress/tensões financeiras) todo o sistema familiar é afetado e fica exposto ao acontecimento stressor (Chichy et al., 2012; Timmer et al., 1996).

No contexto africano, Greef (2000) realizou um estudo com famílias sul-africanas (cf., Tabela 1), com a maioria na fase "saída dos filhos mais velhos" e verificou que determinadas condições funcionam como forças familiares, nomeadamente, a coesão, a adaptabilidade, as competências na resolução dos conflitos conjugais, a satisfação com a qualidade do tempo passado em conjunto e em atividades separadas, a demonstração de afeto existente entre o casal e o relacionamento sexual, o bom relacionamento interpessoal com pessoas externas à família e a comunicação efetiva entre o casal. Estes dados são convergentes com os obtidos por Koen, Van Eeden e Rothmann (2012) num estudo qualitativo com famílias com filhos adolescentes 
etnicamente/culturalmente diversas (cf., Tabela 1). No entanto, Greef (2000) excluiu dos seus estudos as famílias negras, e Koen et al. (2012), apesar de alargar o seu foco para um grupo mais diverso e pouco estudado, basearam-se numa amostra muito reduzida (cf., Tabela 1 ).

\section{Estudos sobre os rituais no ciclo evolutivo das famílias afro-americanas e africanas}

A pesquisa bibliográfica sobre os rituais ao longo do desenvolvimento familiar das famílias africanas identificou dois estudos que apontam para a celebração de rituais tradicionais ou religiosos enquanto marcadores da passagem de uma etapa do ciclo vital para a seguinte.

No artigo de Igreja e Lambranca (2009) destaca-se, para o contexto Moçambicano, a celebração de cerimónias associadas aos valores tradicionais das famílias (i.e., rituais de nascimento, atribuição do nome ao recém-nascido, os ritos de iniciação feminina e masculina, os casamentos e os funerais). Por sua vez, McAdamsMahmoud (2005) apurou que as famílias muçulmanas sunitas de origem africana residentes nos Estados Unidos da América realizavam os seguintes rituais: a) circuncisão dos rapazes; b) o Aqueeqah, que consiste na cerimónia de atribuição do nome ao recém-nascido, normalmente sete dias após o nascimento, sendo escolhido um nome árabe de valores ou características que os pais ambicionam para os filhos c) casamento público, com a estipulação do pagamento do dote; d) e os funerais, realizados preferivelmente antes do pôr-do-sol do dia em que a pessoa morreu (cf., Tabela 1).

Ao contrário da literatura Ocidental que defende uma menor participação nos rituais familiares por parte dos adolescentes e um aumento do tempo passado fora de casa (cf., Eaker \& Walters 2002), nos artigos analisados verificou-se que durante esta fase da adolescência, as famílias tradicionalistas parecem ser muito ritualizadas. A adolescência dos filhos caracteriza-se pela celebração dos rituais de iniciação que, para além de facilitar a integração dos jovens na comunidade têm como objetivo fundamental instruí-los sobre os papéis de género (i.e., responsabilidades inerentes às tarefas domésticas para as mulheres e o papel de provedor do lar para os homens) que lhes vão ser socialmente exigidos (Igreja \& Lambranca, 2009).

Dois estudos realçam a importância dos rituais enquanto elementos organizadores e facilitadores da coesão familiar: o estudo realizado por Júlio (2012) em Angola e o estudo de Chadilha et al., (2005) com uma amostra Afro-Americana (cf., Tabela 1). Enquanto os resultados de Júlio (2012) apontam para a perceção de maior ritualização em famílias com filhos na escola, uma fase que origina 
grandes transformações no seio familiar e a necessidade de ajustamentos globais (Relvas, 1996), a pesquisa de Chadilha et al. (2005) demonstra o papel das reuniões familiares na manutenção dos encontros entre os elementos jovens e idosos das famílias avaliadas.

\section{Os estudos em torno das rotinas no ciclo evolutivo das famílias} afro-americanas e africanas

Dos 22 artigos revistos, seis focam-se nas rotinas familiares. Três artigos demonstram que existem semelhanças entre as famílias com filhos em idade pré-escolar, pertencentes a diversos grupos étnicos, relativamente ao tipo de atividades que realizam, nomeadamente, as atividades fora (e.g., passeios, fazer compras) e dentro de casa (e.g., ver televisão com os filhos, rituais de deitar, partilha de refeições, leitura para os filhos) (cf., Tabela 1; Bradley, Pennar, \& Glick, 2014; Ferreti, 2011; Goede, 2012). No entanto, os grupos diferenciam-se entre si quanto à frequência destas atividades, com as famílias afro-americanas a relatarem, em média, mais atividades realizadas com os seus filhos ao ar livre, mas menor frequência de rotinas de deitar, de leitura e de realização de brincadeiras entre pais e crianças, quando comparadas com as americanas oriundas da Europa e da Índia (Bradley et al., 2014; Ferreti, 2011).

Goede (2012) num estudo qualitativo com dez famílias de raça mista (coloured people) residentes na África do Sul, na etapa de "família com filhos pequenos", sublinhou o papel que as rotinas desempenham, nomeadamente: a) providenciarem uma oportunidade para os elementos da família passarem tempo juntos; b) criarem condições para o desenvolvimento dos filhos; e c) contribuir para o ajustamento do subsistema parental a esta nova fase da vida familiar (cf., Tabela 1). Churchill e Stoneman (2004) na sua pesquisa com uma amostra predominantemente afro-americana (cf., Tabela 1) encontraram uma relação fraca entre as características demográficas e as rotinas realizadas no seio das famílias estudadas, verificando-se uma forte influência de algumas características individuais da mãe (e.g., personalidade, depressão). Os autores verificaram que as meninas têm uma maior probabilidade de se envolverem em rotinas, e que a presença ou ausência de rotinas pode contribuir para o desenvolvimento global das crianças.

A maior parte dos estudos revistos focam-se nas rotinas realizadas por famílias com filhos em idade pré-escolar, o que é convergente com a literatura sobre as rotinas com outras populações, e que aponta para uma maior frequência e envolvimento em rotinas familiares quando os filhos se encontram nesta fase de desenvolvimento (Fiese et al., 2002; Spagnola \& Fiese, 2007). 


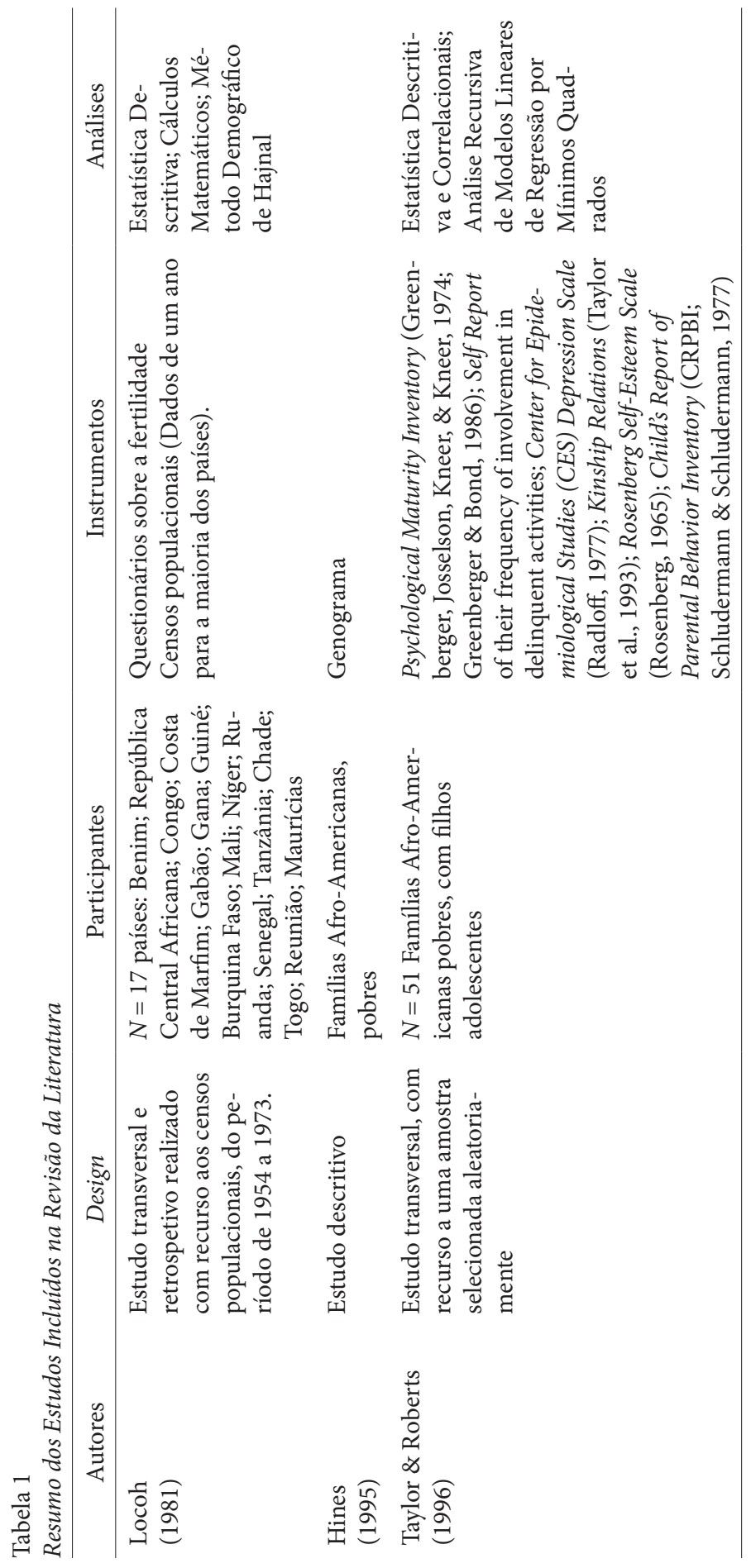




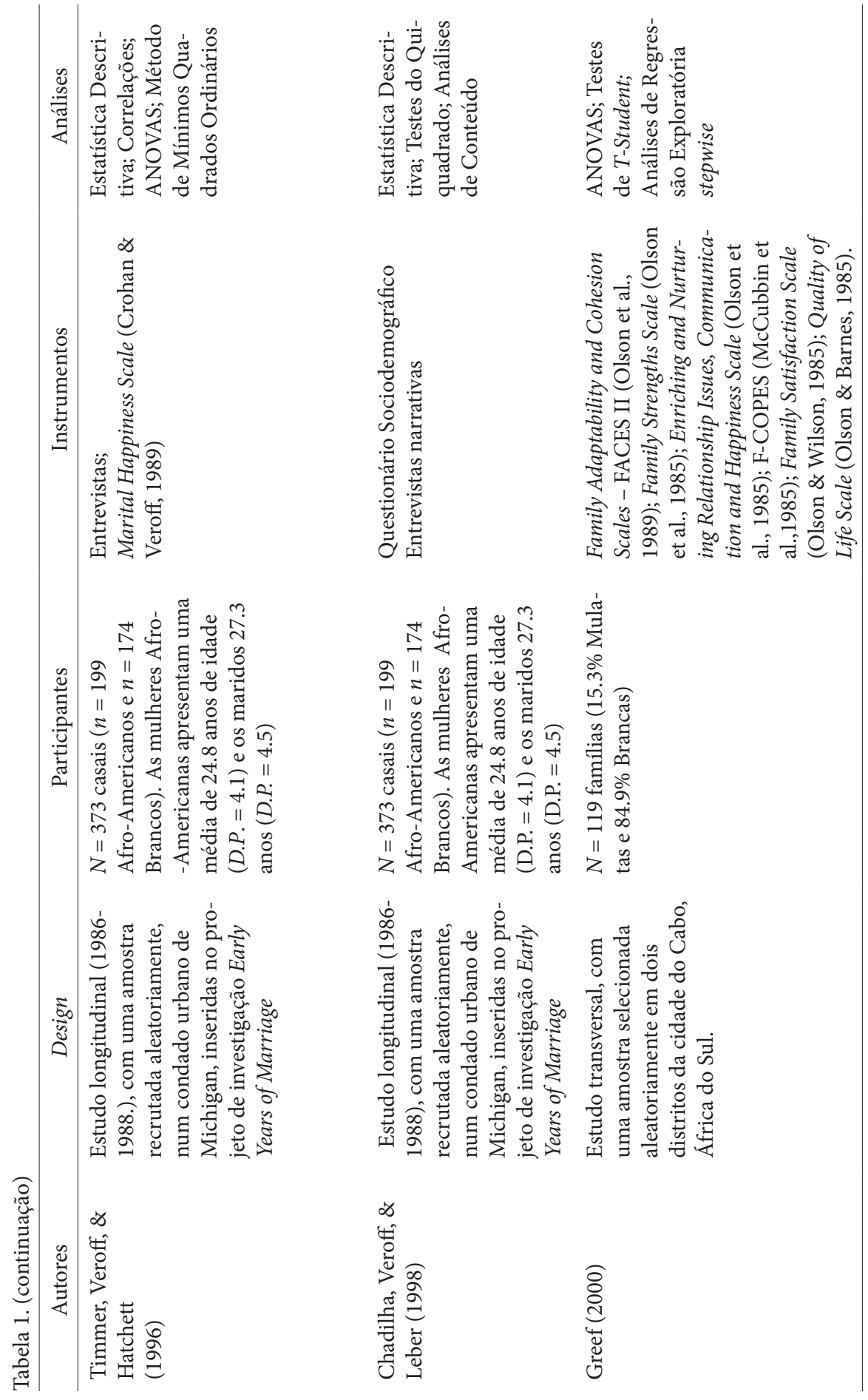




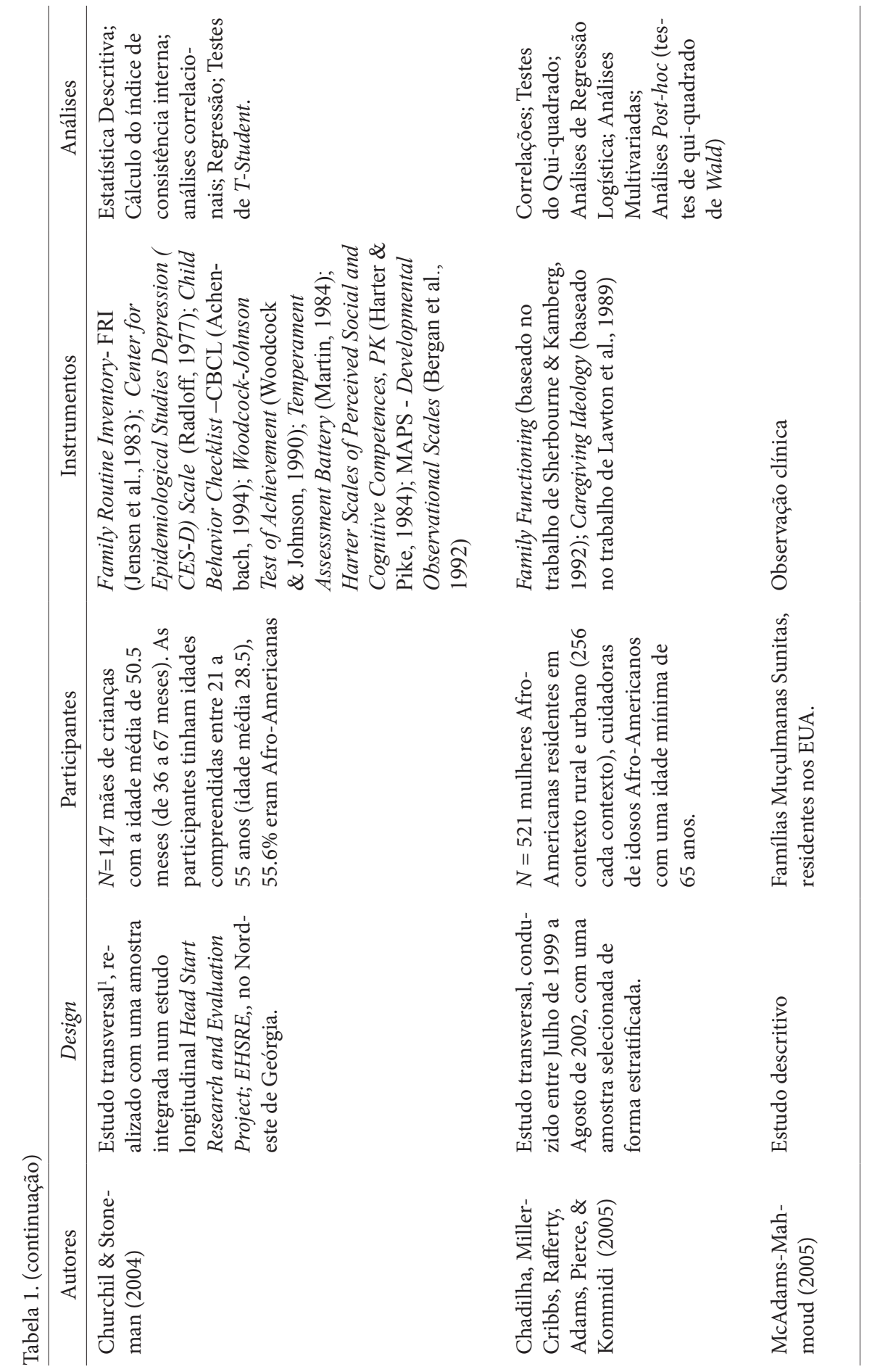




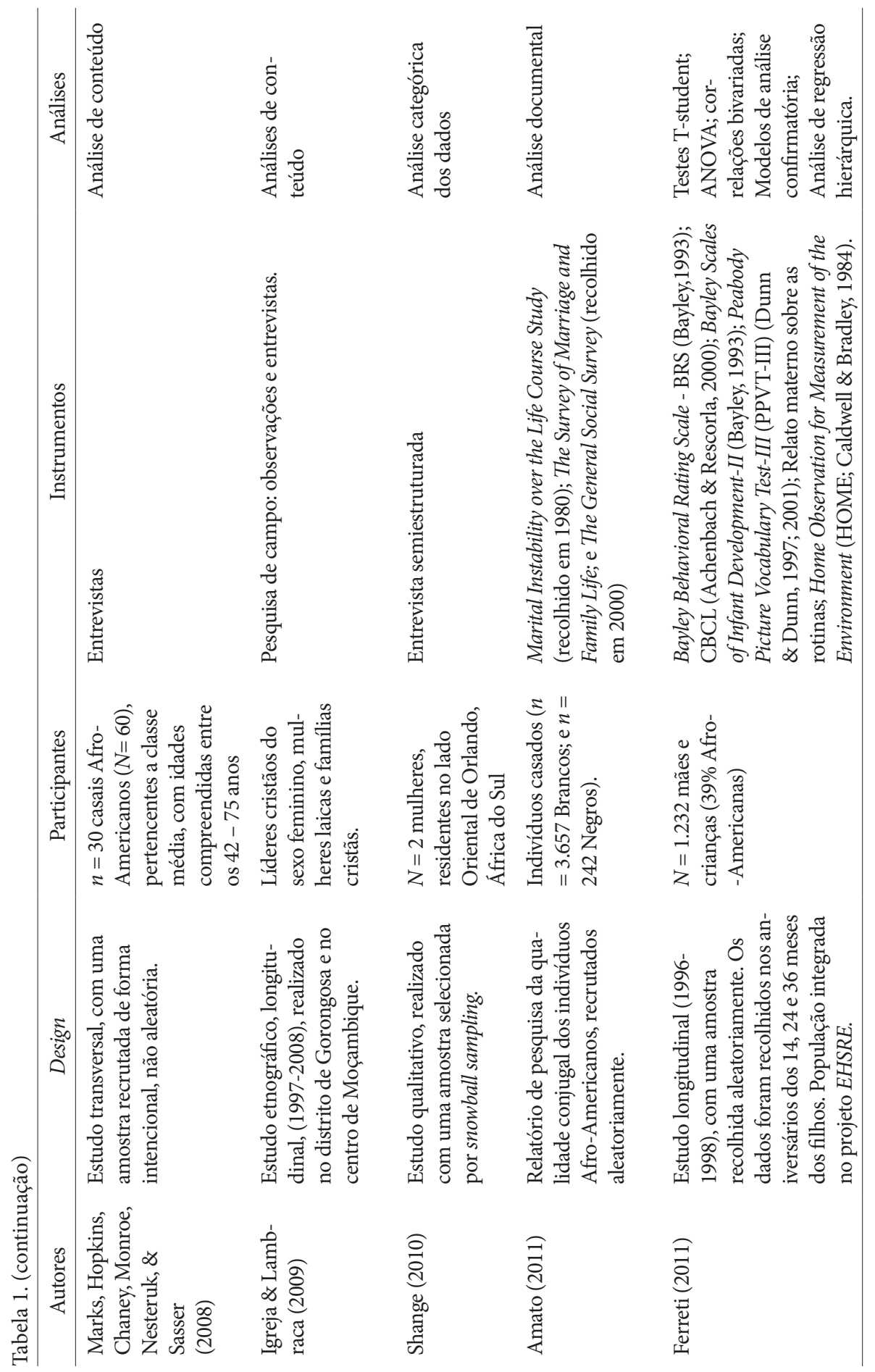




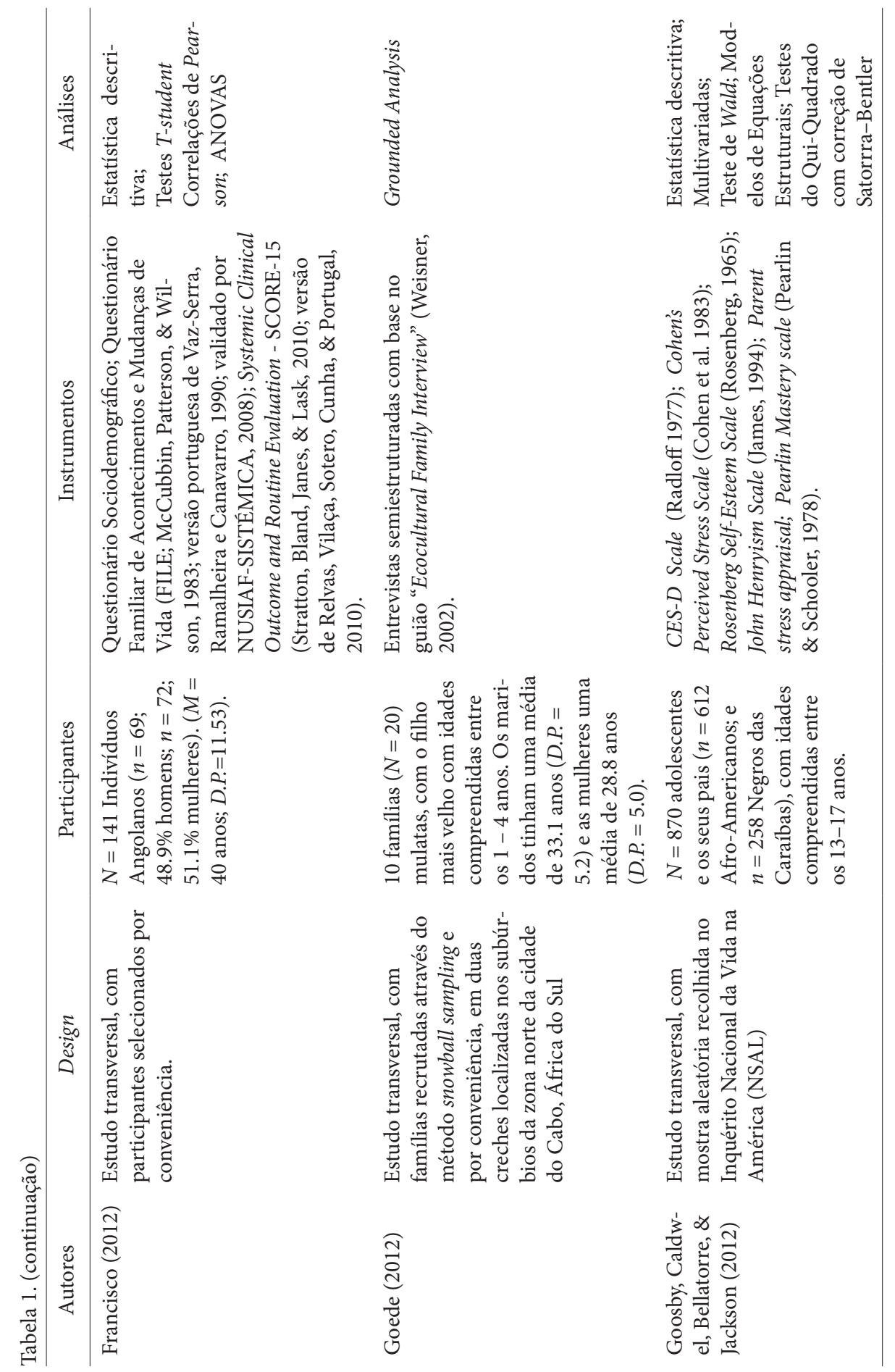




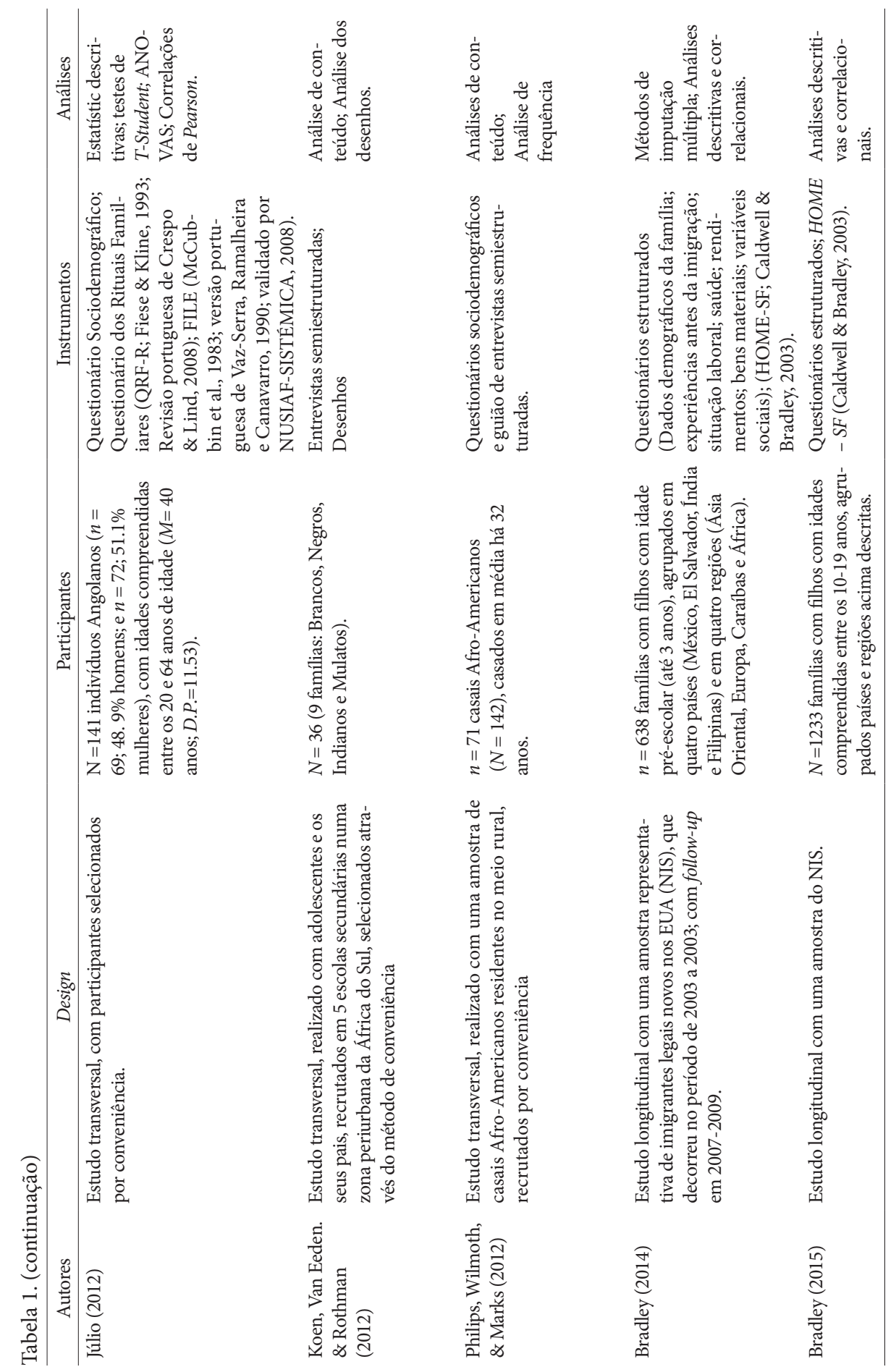




\section{CONCLUSÃO}

A revisão da literatura permitiu ter uma perspetiva sobre os modelos do ciclo vital de famílias africanas (e afro-americanas), onde é realçada a influência da etnicidade/cultura na composição familiar, no desenvolvimento e no funcionamento dos sistemas familiares. As referências consultadas contêm informação que conduz ao questionamento da utilização generalizada de modelos concebidos para as famílias brancas nucleares intactas do Ocidente para as famílias originárias de outros contextos étnicos/culturais, particularmente as de origem africana.

$\mathrm{Na}$ consulta bibliográfica realizada encontrou-se um único modelo do ciclo vital da família africana (Locoh, 1981) mas que se foca exclusivamente em aspetos demográficos do desenvolvimento familiar e na díade mãe-filho, limitando a compreensão da evolução familiar e, concomitantemente, do funcionamento familiar a nível das tensões, das forças, dos rituais e das rotinas.

Verificou-se que nas amostras afro-americanas, a pesquisa revela a necessidade de se conceberem modelos do ciclo vital mais inclusivos que abranjam famílias pertencentes a diferentes níveis socioeconómicos, enquanto no contexto africano prevalece a necessidade de construir modelos do ciclo vital fundamentados na abordagem sistémica do desenvolvimento familiar, que permitam compreender quais são os principais stressores que as famílias enfrentam em cada uma das etapas do ciclo vital, os rituais de transição, bem como as forças e as rotinas que possibilitam o funcionamento familiar ajustado (Relvas, 1996) e enquadrando as especificidades étnicas e tribais que caracterizam as sociedades africanas (e.g., tipologias familiares).

A análise dos artigos sobre o funcionamento familiar permitiu verificar que os indivíduos afro-americanos relatam, ao longo do seu desenvolvimento, situações específicas de stress/tensões familiares (e.g., o ter de lidar e providenciar apoio à família alargada, com ou sem laços sanguíneos). Observou-se, igualmente, que as famílias utilizam forças semelhantes às identificadas por Defrain (1999), contudo destacam-se algumas forças familiares que são específicas destes grupos étnicos (e.g., valores coletivistas). Outro aspeto a destacar, é que os stressores frequentemente relatados pelas famílias americanas e europeias (brancas), como por exemplo as tensões resultantes dos movimentos de entrada e saída da família e os momentos de transição (Olson et al., 1989) não se mostraram relevantes nas amostras africanas e afro-americanas dos estudos revistos.

Apesar de se observar alguma transversalidade ao nível dos fatores de stress e de forças nos vários estudos com famílias africanas e afro-americanas, nenhum estudo revisto procurou avaliar essas variáveis ao longo da trajetória desenvolvimental da família. Por isso, torna-se necessário a identificação dos fatores de stress e de forças familiares nas diferentes etapas do ciclo vital a partir de um modelo construído a partir das particularidades das famílias africanas. 
Os rituais e as rotinas apresentam-se como elementos mediadores dos processos de estabilização, organização, adaptação e coesão familiar. Verificou-se que, para além da etnia/cultura, a religião parece desempenhar um papel importante ao nível dos rituais realizados (McAdams-Mahmoud, 2005). Nos estudos revistos, as rotinas familiares foram mais frequentemente o foco da investigação, possivelmente por serem as mais presentes na vida familiar (Fiese et al., 2002). Tal como se verificou para as tensões e as forças familiares, também os estudos sobre os rituais e as rotinas das famílias africanas são escassos e não integram o percurso desenvolvimental da família, pelo que se torna necessário o recurso a estudos mistos (com questionários de autorrelatos, entrevistas semiestruturadas e registos de observação) para um maior conhecimento das rotinas realizadas em diferentes etapas do ciclo vital e da influência dos rituais no funcionamento familiar.

O presente artigo apresenta algumas limitações: 1) Não se acedeu diretamente aos estudos realizados sobre os primeiros modelos do ciclo vital da família, os de Loomis, Rowntree, Duvall, Hill e Rodgers, citados em Aldous (1990), Carter e McGoldrick (1995) e Relvas (1996); 2) a pesquisa por palavras-chave "etnicidade", "cultura" e "África”, articuladas com "família”, "Ciclo Vital” ou "rituais familiares" como indutores de busca nas bases de referenciação bibliográfica resultaram, essencialmente, em estudos baseados nas experiências pessoais e profissionais dos clínicos ou são realizados no âmbito de outras ciências sociais (Economia e Antropologia) com outras etnias (e.g., da Ásia); 3) a revisão realizada, considerando a escassez de referências sobre famílias africanas englobou os estudos com amostras afro-americanas, podendo ter culminado numa análise dos Africanos e Afro-Americanos de forma monolítica, não especificando as diferenças existentes ao nível do funcionamento familiar inerentes aos valores/ tradições culturais e tribais.

Assim, de futuro será fundamental desenhar projetos de investigação que tenham como objetivo validar empiricamente as propostas teóricas de organização e funcionamento familiar, nomeadamente os modelos do ciclo evolutivo da família, identificando os eventos indutores de stress/tensões, as forças familiares, os rituais e as rotinas em populações africanas de diferentes etnias e culturas

\section{REFERÊNCIAS}

AJ, C. (1998). A study of family life cycle of Korean women. Pogon Sahoe Yongu, 18(1), 56-79.

Aldous, J. (1990). Family development and the life course: Two perspectives on family change. Journal of Marriage and Family, 52(3), 571-583. doi:10.2307/352924 
Amato, P. R. (2011). Marital quality in African American marriages: Research Report. Oklahoma City: National Marriage Resource Center. Consultado em http://www.healthymarriageinfo.org/ resource-detail/index.aspx?rid=3929

Asen, E., \& Tomson, P. (1997). La familia dentro de nosotros: Genogramas. In E. Asen \& P. Tomson (Eds.), Intervención familiar: Guía práctica para los profesionales de la salud (pp. 39-51). Barcelona, Espanha: Paidós.

Bennett, L. A., Wolin, S. J., \& McAvity, K. J. (1988). Family identity, ritual, and myth: A cultural perspective on life cycle transition. In C. J. Falicov (Ed.), Family transitions: Continuity and change over the life cycle (pp. 211-234). New York, NY: The Guilford Press.

Boss, P. (2002). Family stress management: A contextual approach. California, CA: Sage.

Bradley, R. H., Pennar, A., \& Glick, J. (2014). Home environments of infants from immigrant families in the United States: Findings from the new immigrant survey. Infant Mental Health Journal, 35(6): 565-579. doi: 10.1002/imhj.21477

Bradley, R. H., Pennar, A., \& Glick, J. (2015). Home environments of immigrant adolescents in the United States, poster session presented at $14^{\text {th }}$ European congress of Psychology, Milan, Italy.

Carter, B., \& McGoldrick, M. (1995). As mudanças no ciclo de vida familiar: Uma estrutura para a terapia familiar. In B. Carter \& M. McGoldrick (Eds.), As mudanças no ciclo de vida familiar: Uma estrutura para a terapia familiar (pp.16-23). Porto Alegre, Brasil: Artmed.

Chadilha, L. A., Veroff, J., \& Leber, D. (1998). Newlywed's narrative themes: Meaning in the first year of marriage for African American and white couples. Journal of Comparative Family Studies, 29(1), 115-130. Consultado em http://www.jstor.org/stable/41603550

Chadilha, L. A., Miller-Cribbs, J., Rafferty, J., Adams, P., Pierce, R., \& Kommidi, S. (2005). Urban and rural African American female caregivers' family reunion participation. Marriage \& Family Review, 37(1-2), 129-146. doi: 10.1300/J002v37n01_09

Churchill, S. L., \& Stoneman, Z. (2004). Correlates of family routines in head start families. Early Childhood Research and Practice, 6(1). Consultado em http://ecrp.uiuc.edu/v6n1/churchill.html

Cichy, K. E., Stawski, R. S., \& Almeida, D. M. (2012). Racial differences in exposure and reactivity to daily family stressors. Journal of Marriage and Family, 74(3), 572-586. doi: 10.1111/j.1741-3737.2012.00971.x

Defrain, J. (1999). Strong families. Family Matters, 53, 6-13. Consultado em https://aifs.gov.au/publications/family-matters/issue-53/strong-families-around-world

Eaker, D. G., \& Walters, L. H. (2002). Adolescent satisfaction in family rituals and psychosocial development: A developmental systems theory perspective. Journal of Family Psychology, 16(4), 406-414. doi: 10.1037//0893-3200.16.4.406

Ferreti, L. (2011). The influence of family routines on the resilience of low-income preschoolers (Dissertação de mestrado não publicada). Universidade de Auburn, Alabama, Estados Unidos da América.

Fiese, B., Tomcho, T., Douglas, M., Josephs, K., Poltrock, S., \& Baker, T. (2002). A review of 50 years of research on naturally occurring family routines and rituals: Cause for celebration? Journal of Family Psychology, 16(4), 381-390. doi:10.1037//0893-3200.16.4.381

Francisco, A. U. (2012). Acontecimentos de vida e vulnerabilidade ao stress em famílias do sul de Angola (Dissertação de mestrado não publicada). Universidade de Coimbra, Coimbra.

Goede, C. (2012). Family routines during the adjustment and adaptation process of the transition to parenthood (Dissertação de mestrado não publicada). Universidade de Stellenbosch, Cabo Ocidental, África do Sul.

Goosby, B. J., Caldwell, C. H., Bellatorre, A., \& Jackson, J. S. (2012). Ethnic differences in family stress processes among African Americans and black Caribbeans. Journal of African American Studies, 16(3), 406-422. doi:10.1007/s12111-011-9203-0 
Greef, A. P. (2000). Characteristics of families that function well. Journal of Family Issues, 21(8), 948 962. doi:10.1177/019251300021008001

Hill, N. E., Murry, V. M., \& Anderson, V. D. (2005). Sociocultural contexts of African American families. In V. C. McLoyd, N. E. Hill, \& K. A. Dodge (Eds.), African American family life (pp. 21-44).New York, NY: The Guilford Publications.

Hines, P. M. (1995). O ciclo de vida familiar nas famílias negras pobres. In B. Carter \& M. McGoldrick (Eds.), As mudanças no ciclo de vida familiar: Uma estrutura para a terapia familiar (pp. 440465). Porto Alegre, Brasil: Artmed.

Hoffman, L. (1995). O ciclo de vida familiar e a mudança descontínua. In B. Carter \& M. McGoldrick (Eds.), As mudanças no ciclo de vida familiar: Uma estrutura para a terapia familiar (pp. 84-96). Porto Alegre, Brasil: Artmed.

Igreja, V., \& Lambranca, B. D. (2009). The Thursdays as they live: Christian religious transformation and gender relations in postwar Gorongosa, central Mozambique. Journal of Religion in Africa, 39(3), 262-294. Consultado em http://www.jstor.org/stable/20696817

Jiang, Q., Guo, Z., Li, S., \& Feldman, M. W. (2013). The life cycle of bare branch families in China: A simulation study. Canadian Studies in Population, 40(3-4), 134-148. Consultado em http:// www.ncbi.nlm.nih.gov/pubmed/24174704

Júlio, M. (2012). Caraterização dos rituais familiares numa amostra de casais do Sul de Angola (Dissertação de mestrado não publicada). Universidade de Coimbra, Coimbra.

Kauffmann, D. (2000). A família reconstituída e o seu ciclo vital: Uma análise qualitativa de seus perfis (Trabalho de fim de curso). Universidade Presbiteriana Mackenzie, Brasil.

Koen, V., Van Eeden, C., \& Rothmann, S. (2012). An exploration of families' psychosocial wellbeing in a South African context. Journal of Psychology in Africa, 22(3), 343-357. doi: 10.1080/14330237.2012.10820539

Lavee, Y. (2013). Stress processes in families and couples. In Y. Lavee, G. W. Peterson, \& K. R. Bush (Eds.), Handbook of marriage and the family (pp.159-176). New York, NY: Springer Science + Business Media. doi: 10.1007/978-1-4614-3987-5_8

Lind, W. (2008). Casais biculturais e monoculturais: Diferenças e recursos. (Tese de doutoramento não publicada). Universidade de Lisboa, Lisboa.

Locoh, T. (1981, Julho). Demographic aspects on the family life cycle in sub-saharan Africa, Comunicação apresentada no Encontro final sobre a metodologia do ciclo vital da família da Organização Mundial da Saúde, Wiesbaden, Alemanha.

Marks, L. D., Hopkins, K., Chaney, C., Monroe, P. A., Nesteruk, O., \& Sasser, D. D. (2008). “Together, we are strong": A qualitative study of happy, enduring African American marriages. Family Relations, 57(2), 172-185. doi: 10.1111/j.1741-3729.2008.00492.x

McAdams-Mahmoud, V. (2005). African American muslin families. In M. McGoldrick, J. Giordano, \& N. Garcia-Preto (Eds.), Ethnicity and family therapy (pp. 138-152). New York, NY: The Guilford Press.

McCubbin, H. I., \& Patterson, J. M. (1983). The family stress process: The double ABCX model of adjustment and adaptation. In H. I. McCubbin, M. B. Sussman, \& J. M. Patterson (Eds.), Social stress and the family: Advances and developments in family stress theory and research (pp. 7-37). New York, NY: The Haworth Press.

McGoldrick, M. (1993). Ethnicity, cultural diversity, and normality. In F. Walsh (Ed.), Normal Family Process (pp. 331-360). New York, NY: The Guilford Press.

McGoldrick, M. (1995). Etnicidade e o ciclo de vida familiar. In B. Carter, \& M. McGoldrick (Eds.), As mudanças no ciclo de vida familiar: Uma estrutura para a terapia familiar (pp. 65-84). Porto Alegre, Brasil: Artmed. 
McGoldrick, M., Giordano, J., \& Garcia-Preto, N. (2005). Ethnicity and Family Therapy. New York: Guilford Press.

Melo, A. T., \& Alarcão, M. (2011). Avaliação de processos de resiliência familiar: Validade e fidelidade do Questionário de Forças Familiares. Mosaico, 48, 34-41.

Neighbour, R. (1985).The family life-cycle. Journal of the Royal Society of Medicine, 78(8), 11-15. Consultado em http://www.ncbi.nlm.nih.gov/pmc/articles/PMC1289511/

Olson, D., McCubbin, H., Barnes, H., Larsen, A., Muxen, M., \& Wilson, M. (1989). Families: What makes them work. California, CA: Sage Publications.

O'Rand, A. M., \& Krecker, M. L. (1990). Concepts of the life cycle: Their history, meanings and uses in the social sciences. Annual Review of Sociology, 16, 241-262. doi:10.1146/annurev. so.16.080190.001325

Phillips, T. M., Wilmoth, J. D., \& Marks, L. D. (2012). Challenges and conflicts... Strengths and supports: A study of enduring African American marriages. Journal of Black Studies, 43(8), 936-952. doi: 10.1177/0021934712463237

Relvas, A. P. (1996). O ciclo vital da família: Perspectiva sistémica. Porto: Edições Afrontamento.

Shange, G. L. (2010). Exploring the family life cycle from an African perspective (Dissertação de mestrado não publicada). Universidade da África do Sul, Pretória, África do Sul.

Spagnola, M., \& Fiese, B. H. (2007). Family routines and rituals: A context for development in the lives of young children. Infants \& Young Children, 20(4), 284-299. doi: 10.1097/01. IYC.0000290352.32170.5a

Taylor, R. J., Chatters, L. M., Woodward, A. T., \& Brown, E. (2013). Racial and ethnic differences in extended family, friendship, fictive kin, and congregational informal support networks. Family Relations, 62(4), 609-624. doi: 10.1111/fare.12030

Taylor, R., \& Roberts, D. (1996). Kinship support and maternal and adolescent well-being in economically disadvantaged African-American families. Child Development, 66(6), 1585-1597. doi: $10.2307 / 1131898$

Timmer, S., Veroff, J., Hatchett, S. (1996). Family ties and marital happiness: The different marital experiences of black and white newlywed couples. Journal of Social and Personal Relationships, 13(3), 335-359. doi: 10.1177/0265407596133003

Van Rooyen, J., \& Du Plessis, P. J. (2003). A conceptual and empirical validation of the household lifecycle concept in South Africa. South Africa Journal of Industrial Psychology, 29(3), 30-37. Consultado em http://www.sajip.co.za/index.php/sajip/article/viewFile/107/103

Widmer, E. D., \& Gauthier, J. (2013). Cohabitational trajectories and the family life cycle. In R. Levy \& E. D. Widmer (Eds.), Gendered life courses between standardization and individualization: A European approach applied to Switzerland (pp. 53-71). Zurich, Switzerland: LIT. 